\title{
The contemporary oral health care provider
}

\author{
Armando Montesinos Flores* \\ Department of Orthodontics, Division of Postgraduate Studies and Research, Dental Faculty, National University of Mexico, Mexico
}

Health and disease are a duet performing since the beginning of mankind, the ideal homeostatic state of man is normal health, nevertheless every human being has different stages in which this equilibrium state is broken and disease manifests with sequels or not when heath is restored. Someone in the prehistoric community thru the different historic stages, to the complex contemporary society has supervised and applied medicine. In ancient times, the same people that practiced medicine applied oral health care. The first professional European "dentists" were known as barber-surgeons but it is Pierre Fouchard who is credited as being father of "modern dentistry" because his book was the first to describe a comprehensive system for the practice of dentistry including basic oral anatomy and function.

Dentistry expanded rapidly throughout the $20^{\text {th }}$ and $21^{\text {st }}$ century with different programs and specializing in different fields including orthodontics, pediatric dentistry, periodontics, maxillofacial surgery, prosthodontics, implantology, oral radiology, etc. The oral health provider in its different fields practice has also evolved thru the different historical stages because of scientific data evolution, technology development, new philosophies of treatment and different society; people perception of healthcare services is changing constantly.

Today society is quiet a challenge to every healthcare provider, the easy access to knowledge and good or bad information through the Web, makes every patient a doctor or specialist with such authority as a reviewer of a Board case in a given specialty. Patients seek for more than one opinion with different professionals before starting any treatment and pay more attention to the whole process, especially in the final results; as a consequence patients initiate more lawsuits for

Copyright: (C2016 Flores AM. This is an open-access article distributed under the terms of the Creative Commons Attribution License, which permits unrestricted use, distribution, and reproduction in any medium, provided the original author and source are credited. malpractice if unsatisfied with treatment or a bad influence of another healthcare provider.

So, the oral health provider must keep up adapting and changing as the world turns, first of all the professional needs to be updated with the latest scientific data and discoveries in order to implement evidence based practice. Technology innovations need to be integrated into daily practice and make it available to study, diagnose, monitor ant treat patients. The contemporary oral health provider needs to sharpen his communication skills; good interaction between professionals, with the team and patients is crucial. Special attention also needs to be considered to the social media; it is an integral part of everyday life to share information either good or bad one.

And what about the clinician practice? Dentists and specialists are bombed with a variety of products and systems to treat patients, in fact a large group of professionals relies only in the continuing education provided by the commercial companies. Oral health providers must analyze scientific data before accepting every claim of a new or miracle dental product or technique; the popular saying "Knowledge and information is power" is and undisputed truth. If every professional could aim and seek to be a leader instead of a follower, would revolutionize the whole science.

The contemporary oral health provider must seek function, aesthetics, periodontal integrity, dentition stability, healthy teeth, gums and associated structures of the stomatognathic system as treatment goals. Dentistry and its different specialties and fields will continue to evolve; it depends on all professionals to guide its evolution into the right direction.
Correspondence to: Armando Montesinos Flores, Department of Orthodontics, Division of Postgraduate Studies and Research, Dental Faculty, National University of Mexico, Mexico, E-mail: amfortod_@hotmail.com

Received: November 20, 2016; Accepted: December 16, 2016; Published: December 19, 2016 